\title{
PADRÕES DE DISTRIBUIĈ̃O E OCORRÊNCIA DO ICTIOPLÂNCTON NO MÉDIO RIO XINGU, BACIA AMAZÔNICA, BRASIL
}

RESUMO: O estudo teve como objetivo analisar a abundância e distribuição do ictioplâncton na região da 'Volta Grande do Xingu', Pará, Brasil. As coletas foram realizadas em 04 campanhas durante as fases do ciclo hidrológico local, distribuídas em 16 estações de amostragens. Foram capturados 95 ovos e 398 larvas de peixes, classificadas em 25 táxons, pertencentes a 05 ordens, 16 famílias, 19 gêneros e 18 espécies, dentre as quais 09 são utilizadas como fonte de renda, alimento e subsistência da população local. A maior captura de ovos e larvas foi observada no período noturno, especialmente em locais próximos a bancos de areia, boca de lagos e ao longo dos tributários. Os dados evidenciaram um padrão sazonal, em que a elevada abundância do ictioplâncton esteve relacionada ao pulso de inundação e a pluviosidade local, as demais variáveis ( $\mathrm{T}^{\circ}, \mathrm{pH}, \mathrm{OD}$ e condutividade) também influenciaram de forma diferentemente a distribuição do ictioplâncton. Os resultados revelaram que a área estudada é utilizada como área de desova, criação e dispersão de ovos e larvas de uma variedade de espécies de peixes, evidenciando a necessidade de sua preservação.

PALAVRAS-CHAVE: Amazônia Oriental; Ovos e larvas; Peixes; Preservação; Variáveis ambientais.

\section{DISTRIBUTION AND OCCURRENCE PATTERNS OF ICHTHYOPLANKTON IN THE MIDDLE RIVER XINGU, AMAZON BASIN, BRAZIL}

ABSTRACT: Abundance and distribution of ichthyoplankton in the Volta Grande do Xingú, Pará, Brazil, are analyzed. Collections were undertaken during four campaigns in the local hydrological cycle phases, distributed into 16 sampling stations, featuring

\footnotetext{
Doutor em Ciência Animal pela Universidade Federal do Pará (UFPA); Docente do curso de Engenharia de Pesca, Instituto de Ciências e Tecnologia das Águas da Universidade Federal do Oeste do Pará (UFOPA), Santarém (PA), Brasil; E-mail: dmzacardi@hotmail.com

** Graduada em Engenharia de Pesca pelo Instituto de Ciências e Tecnologia das Águas da Universidade Federal do Oeste do Pará (UFOPA), Santarém (PA), Brasil.
} 
95 eggs and 398 larvae of fish of 25 taxa belonging to 5 orders, 16 families, 19 genera and 18 species, of which 9 were the source of income, food and subsistence of the local population. Largest egg and larvae collection occurred during the night period, especially in places close to sand banks, lake entrances and along the tributaries. Data revealed a seasonal pattern where high ichthyoplankton abundance was related to the flood pulse and rainfall. The other variables (temperature, $\mathrm{pH}$, OD and conductivity) also affected ichthyoplankton distribution differently. Results showed that the area studied is a spawning, breeding and egg and larvae dispersion of several fish species requiring preservation.

KEY WORDS: Eggs and larvae; Fish; Environmental variables; Preservation; Eastern Amazon.

\section{INTRODUÇÃO}

$\mathrm{O}$ rio Xingu pertence ao sistema fluvial amazônico, sendo classificado como rio de água clara pobre em sedimento, o que confere boa transparência às suas águas, podendo, entretanto, em alguns de seus trechos, ser rico em matéria dissolvida (CAMARGO; GHILARDI-JÚNIOR, 2009). Está entre os principais afluentes da margem direita do rio Amazonas, desembocando nas proximidades da cidade de Porto de Moz (PA), aproximadamente $300 \mathrm{~km}$ a leste de Santarém e a $400 \mathrm{~km}$ da cidade de Belém, possui como característica alternâncias bruscas de altitudes em seu fluxo longitudinal.

No trecho médio, situa-se uma área denominada de 'Volta Grande do Xingu', em que o nível do rio desce cerca de $70 \mathrm{~m}$ de altura em todo o seu percurso, característica de perfil topográfico que oferece um dos maiores potenciais hidráulicos do Baixo Amazonas (SALOMÃO et al., 2007), que, atualmente, abriga a construção da Usina Hidrelétrica de Belo Monte, com futuro potencial hidrelétrico estimado de 11.187 mil megawatts (MW) e terá um reservatório estimado de $440 \mathrm{~km}^{2}$ (LUCAS et al., 2009).

Os grandes rios exercem um importante papel no desenvolvimento da sociedade humana e estão entre os ecossistemas mais intensamente fragmentados, sendo que a maioria deles por barragens (JAGER et al., 2001). Este tipo de impacto 
exibe um potencial de modificação nos atributos físicos, químicos e biológicos do sistema fluvial, podendo alterar a composição e abundância da diversidade de peixes (AGOSTINHO; JÚLIO-JUNIOR; BORGHETTI, 1992; BENEDITO-CECÍLIO; AGOSTINHO, 2000), interferir significativamente na dinâmica dos organismos planctônicos e dos nutrientes (MEDEIROS, 2006), além de reduzir ou impedir a comunicação do rio com as várzeas alterando o fluxo natural e, consequentemente, restringindo as funções ecológicas dos ecossistemas aquáticos (AGOSTINHO; PELICICE; GOMES, 2008).

Portanto, torna-se fundamental compreender o papel deste trecho médio do rio Xingu e seus tributários, para o ciclo inicial de desenvolvimento dos peixes, antes e depois do barramento, sendo importante para o entendimento dos processos de ocupação desses elementos da ictiofauna regional, uma vez que a pesca na região constitui uma das atividades mais importantes e a principal fonte de alimento proteico local. Esse valor econômico e social dos recursos pesqueiros existentes nessa região é inestimável, não somente pela extensão da bacia hidrográfica, mas também pela riqueza de espécies destes ambientes, sendo que em alguns grupos há a presença de uma intensa exploração pela pesca ornamental e comercial (CAMARGO et al., 2009; CARVALHO-JÚNIOR et al., 2011).

Sabe-se que grande parte dos peixes de importância ecológica e de interesse comercial, como os Characiformes e diversos Siluriformes, possuem suas atividades reprodutivas ligadas à dinâmica fluviométrica. $\mathrm{O}$ aumento do nível fluviométrico contribui na elevação do número de indivíduos recém-eclodidos, que ficam à deriva na calha dos rios no período de enchente e que diminuem em abundância nos períodos subsequentes (LIMA; ARAÚJO-LIMA, 2004). A compreensão destas variações é fundamental para o entendimento da abundância e distribuição dos recursos pesqueiros.

Contudo, na bacia amazônica existem poucos estudos sobre ictioplâncton e estão concentrados, principalmente, em ecossistemas de água branca, no sistema Solimões/Amazonas. No entanto, são escassas as informações sobre o ictioplâncton em ecossistemas amazônicos de águas pretas e claras, limitando-se aos trabalhos de Lima e Araújo-Lima (2004) e Oliveira e Ferreira (2008).

Neste contexto, a proposta do trabalho foi conhecer a composição, abundância e a distribuição do ictioplâncton presente em distintas unidades de 
hábitat, no trecho médio do rio Xingu, ampliando assim, o conhecimento ecológico e biológico desse ecossistema. Os dados poderão subsidiar a implantação de políticas de manejo e estratégias de conservação para preservação das espécies de peixes desta importante bacia.

\section{MATERIAL E MÉTODOS}

\section{1 ÁREA DE ESTUDO}

O estudo da comunidade ictioplanctônica abrangeu a área da "Volta Grande do Xingu" entre as coordenadas $03^{\circ} 32^{\prime}$ '44,7" S; $051^{\circ} 57^{\prime} 46,0^{\prime \prime}$ W e $03^{\circ} 366^{\prime} 54,1^{\prime \prime}$ $S$; $051^{\circ} 49^{\prime} 24,7^{\prime \prime}$ W, no município de Senador José Porfírio, no Estado do Pará (Figura 1). As coletas foram realizadas em 16 estações de amostragens, devidamente georreferenciadas, nos meses de setembro e dezembro de 2014 e abril e junho de 2015, na zona fluvial do rio Xingu, rio Ituna e rio Itatá.

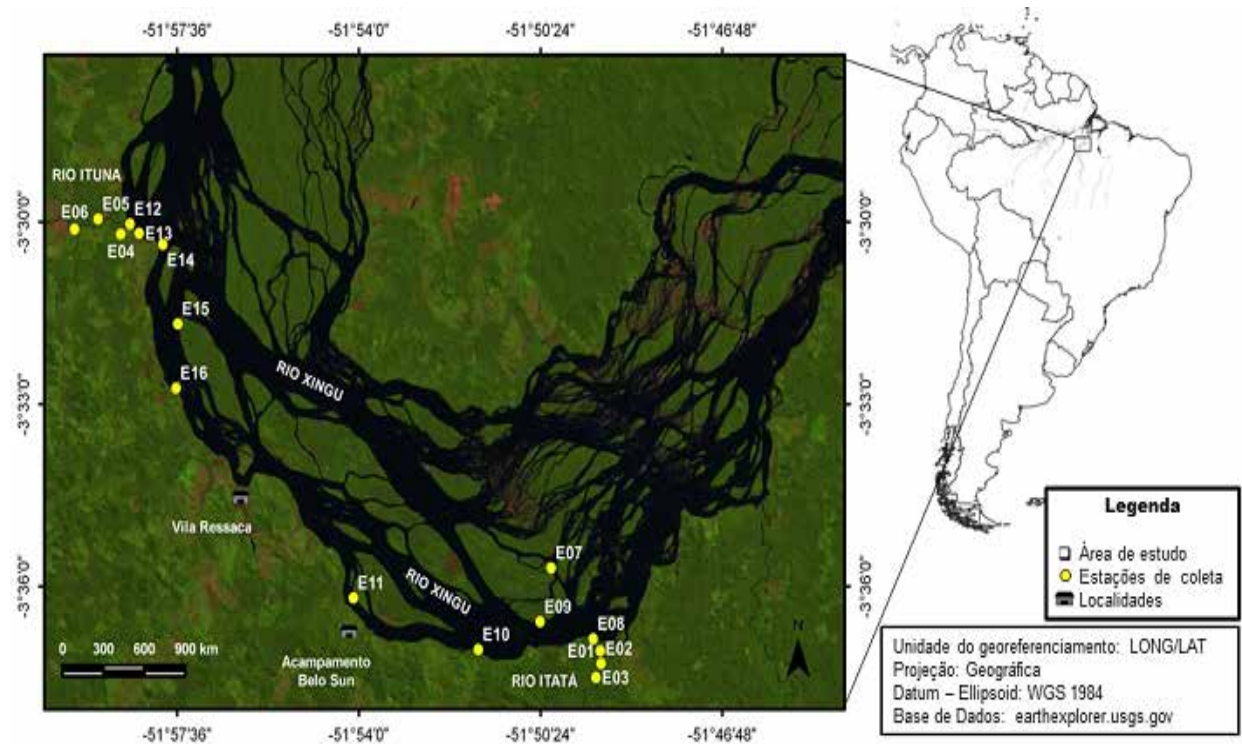

Figura 1. Localização da Volta Grande do Xingu, com destaque para as 16 estações de amostragem de ictioplâncton (rio Itatá - E01, E02 e E03; rio Ituna - E04, E05 e E06; e rio Xingu - E07, E08, E09, E10, E11, E12, E13, E14, E15 e E16), no trecho médio do rio Xingu, Pará, Brasil. 
O rio Xingu possui uma extensão de $1.500 \mathrm{~km}$, desde suas nascentes no Planalto Central Brasileiro até sua foz no rio Amazonas, e drena uma área de 540.000 $\mathrm{km}^{2}$, banha áreas de substrato rochoso, sendo caracterizado por águas claras, ligeiramente ácidas, com alta transparência e concentrações de oxigênio dissolvido, baixa carga de material em suspensão e fontes de material orgânico (CAMARGO, 2004).

O trecho médio do rio Xingu caracteriza-se por um clima quente, segundo a classificação climática de Köppen corresponde a um clima tropical e predominantemente úmido (Am), com temperatura média anual oscilando entre 17,5 a $24,5{ }^{\circ} \mathrm{C}$ e umidade relativa entre 84 a $86 \%$. A precipitação média anual varia entre $2.066 \mathrm{~mm}$ a $2.379 \mathrm{~mm}$. O período de chuvas locais inicia-se em novembro, com maior intensidade de dezembro a maio. Essa variação no regime de chuvas incide na vazão do rio, que apresenta valores médios de 8.000 a $10.000 \mathrm{~m}^{3} . \mathrm{s}^{-1} \mathrm{e}$ diminui até $2.000 \mathrm{~m}^{3} . \mathrm{s}^{-1}$ durante a estiagem (junho a outubro). Estas variações sazonais e interanuais determinam números significativos de vazão, sendo a vazão média anual, na área estudada, de aproximadamente $7.851 \mathrm{~m}^{3} \cdot \mathrm{s}^{-1}$ (GHILARDI-JÚNIOR; CAMARGO, 2009).

\subsection{COLETA E PROCESSAMENTO DO MATERIAL BIOLÓGICO}

As coletas ocorreram em quatro campanhas sazonais vinculadas às fases do ciclo hidrológico (Figura 2): Campanha 1 durante a fase de seca do rio Xingu - setembro de 2014 (C1); Campanha 2 durante a enchente - dezembro de 2014 (C2); Campanha 3 durante a cheia - abril de 2015 (C3); e Campanha 4 durante a vazante - junho de 2015 (C4), em ciclos de amostragem diurno e noturno, para tentar evidenciar variações circadianas, perfazendo 32 amostras por campanha, totalizando 128 amostras no final do estudo. 


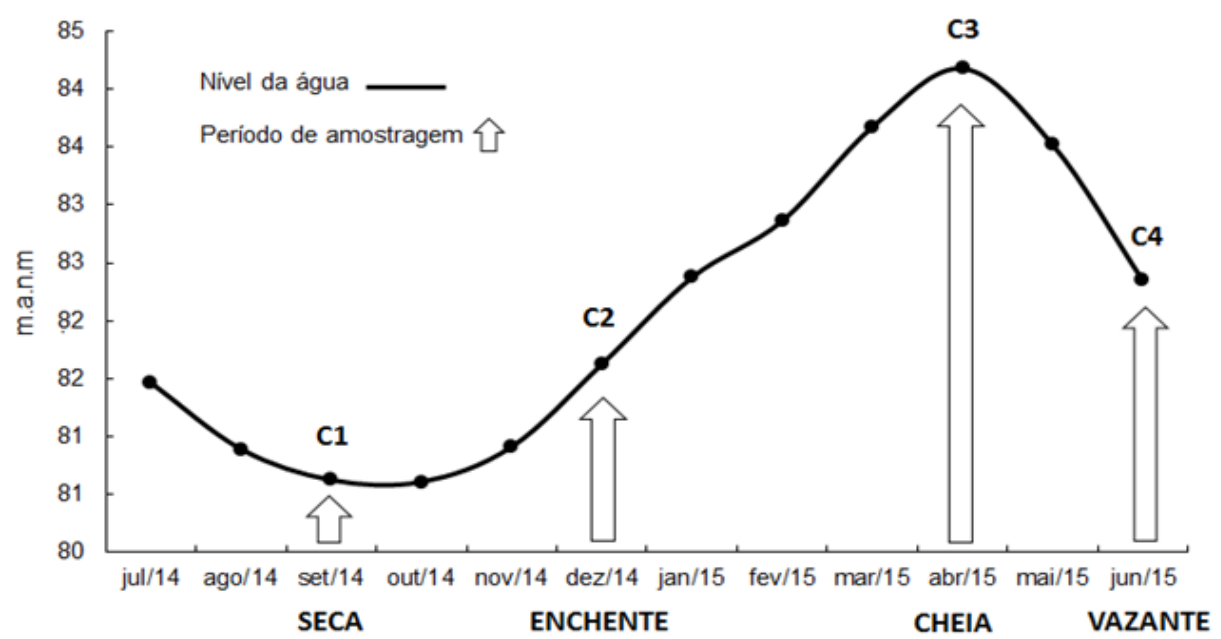

Figura 2. Variação do nível da água do rio Xingu durante o ciclo hidrológico de julho de 2014 a junho de 2015, com a indicação das campanhas (C1, C2, C3 e C4) e períodos de amostragem (m.a.n.m. = metros acima do nível do mar).

As estações de amostragem selecionadas abrangeram locais diversificados como: Confluência de rios - áreas caracterizadas pela ligação da descarga das águas mais turvas dos tributários com o rio Xingu, constitui-se no ponto de mudança no fluxo, na quantidade de sedimento transportado e na hidrodinâmica (Figura 3 A); Canal central dos rios - apresenta constante fluxo de correntes de água com maior carga de partículas em suspensão, que determinam menor transparência quando comparado ao rio Xingu (Figura $3 \mathrm{~B}$ ); Áreas próximas a barrancos - regiões muito turbulentas e caracterizadas por forte processo de erosão do solo das margens e pelo transporte (ou carregamento) de seus sedimentos rio abaixo (Figura $3 \mathrm{C}$ ); Áreas próximas a bancos de areia - constituídas por faixas de deposição de sedimentos ao longo dos rios, em áreas pouco turbulentas, com baixa profundidade e em constante dinâmica, modificando continuamente a sua posição em relação ao canal do rio (Figura 3 D); Áreas próximas a pedral - trecho rochoso no leito do rio Xingu, caracterizado por apresentar grande correnteza e oxigenação das águas (Figura $3 \mathrm{E}$ ); Bocas de canais de lagos - conectam as águas do rio com os lagos. Alguns destes canais secam completamente durante o período de seca, tornando alguns lagos completamente isolados do rio principal (Figura $3 \mathrm{~F}$ ). 


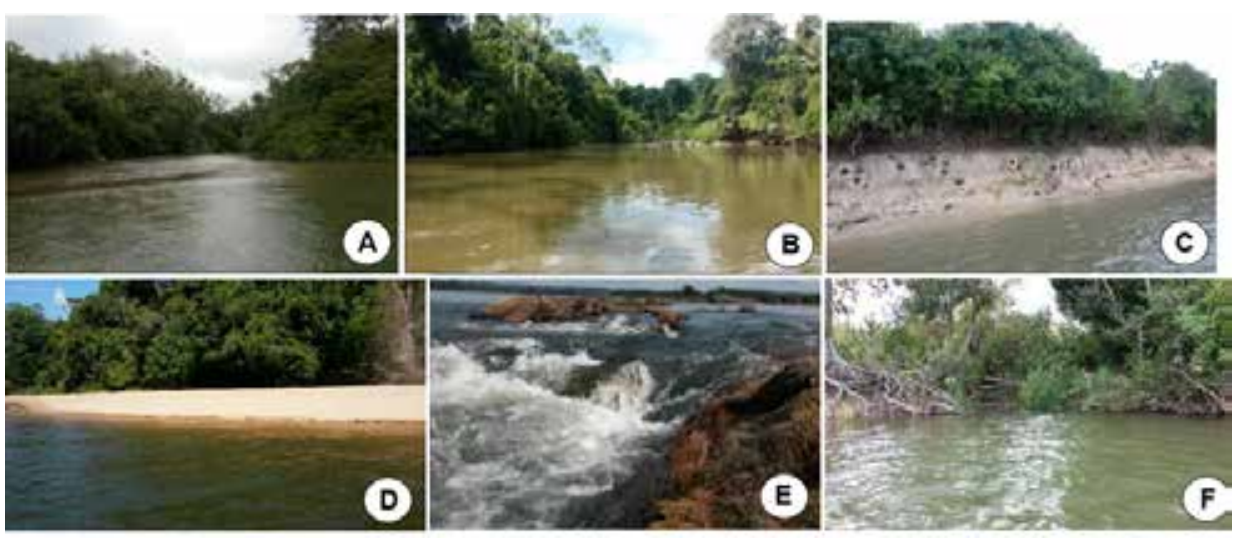

Figura 3. Estações de amostragem no trecho médio da Volta Grande do Xingu, Pará. A - Estação próxima à confluência de rios; B - Estação no canal central do rio Itatá; C - Estação próxima a barrancos no rio Xingu; D - Estação próxima a bancos de areia no rio Xingu; $\mathbf{E}$ - Estação próxima aos pedrais no leito do rio Xingu; e F - Estação próxima à confluência de canal de lago com o rio Xingu.

Para obtenção do material biológico foi utilizada rede de plâncton cônicocilíndrica, constituída de malha de $300 \mu \mathrm{m}$ com abertura de boca de $50 \mathrm{~cm}$ e $1,50 \mathrm{~m}$ de comprimento, dotada de fluxômetro mecânico no centro da boca da rede, para registro do volume de água filtrada durante os arrastos horizontais realizados na subsuperfície da coluna d'água com o auxílio de uma embarcação. Após a coleta, as amostras foram fixadas em solução formalina a $10 \%$ e acondicionadas em frascos de polietileno de $500 \mathrm{ml}$ devidamente etiquetados.

As variáveis ambientais como a temperatura da água, condutividade elétrica, potencial hidrogeniônico e oxigênio dissolvido foram registradas in loco, utilizandose medidor multiparâmetro portátil digital (Hanna HI - 9828). Os valores dos índices pluviométricos e níveis fluviométricos utilizados para as análises foram cedidos pela Belo Sun Mineração Ltda. que fica situada à margem direita do trecho estudado.

Em laboratório, o material biológico foi triado, separando ovos e larvas de peixes do material em suspensão, detritos e do plâncton total, com auxílio de pinças, placas de Petri e microscópio estereoscópico. Em seguida o material foi quantificado e as larvas identificadas ao menor nível taxonômico possível, com base em características morfológicas, merísticas e morfométricas com auxílio de bibliografias especializadas: Araújo-Lima (1994); Araújo-Lima e Donald (1988); Leite et al. (2007); Nascimento e Araújo-Lima (1993); Nascimento e Araújo-Lima (2000); Nakatani et al. (2001). 
Essa técnica consiste na identificação a partir de uma sequência de indivíduos em diferentes estágios, desde larvas em estágios mais avançados até larvas recémeclodidas, tendo como base alguns caracteres morfológicos e merísticos como a forma do corpo, o padrão de pigmentação, sequência de formação das nadadeiras, a posição relativa da abertura anal em relação ao corpo, formato do trato digestivo e da bexiga natatória, número de miômeros, de raios e espinhos das nadadeiras. As larvas consideradas não identificadas corresponderam a indivíduos com estruturas danificadas e/ou estágio muito inicial de desenvolvimento (recém-eclodidas).

O desenvolvimento larval foi dividido em quatro estágios: larval vitelino, pré-flexão, flexão e pós-flexão, baseando-se no grau de flexão da seção terminal da notocorda, durante o desenvolvimento da nadadeira caudal e seus elementos de suporte, assim como proposto por Nakatani et al. (2001). Os espécimes identificados foram armazenados em frascos de vidro etiquetados contendo formalina a $4 \%$.

\subsection{ANÁLISE DOS DADOS}

Para estimativas da densidade, as amostras foram padronizadas para um volume de $10 \mathrm{~m}^{3}$, de água filtrada, baseado em Nakatani et al. (2001). Os padrões de distribuição foram posteriormente analisados pela variação da densidade das espécies, nas campanhas de coleta e nas estações amostradas. Os dados de densidades médias de ovos e larvas foram previamente transformados em $\log 10(\mathrm{x}+1)$.

Para verificar diferenças significativas na densidade média na distribuição espacial (estações de amostragem), temporal (período diurno e noturno) e sazonal (campanhas de amostragem associadas ao pulso de inundação) do ictioplâncton, aplicou-se ANOVA bifatorial considerando as estações e campanhas como fatores independentes, utilizando o software STATISTICA 7.0. Os pressupostos da ANOVA foram testados pelo teste de Shapiro-Wilk para normalidade e pelo teste de Levene para homogeneidade das médias dos dados (PETERS, 1986). Quando a Análise de Variância foi significativa, aplicou-se o teste pos hoc de Tukey para verificar qual variável diferiu das demais. Diferenças significativas implicam em $p<0,05$.

Para a avaliação da variação dos parâmetros ambientais e sua relação com a densidade de larvas e ovos de peixes foram realizadas correlações de Pearson entre os escores dos eixos da análise fatorial em componentes principais (Análise dos Componentes Principais - ACP) utilizando os dados logtransformados, a fim de reduzir a influência da dominância de poucas espécies em detrimento de outras. 
Assim, foram retidos para a interpretação os eixos com autovalores maiores que 1,0, segundo o critério de Kaiser-Guttman (JACKSON, 1993); esta análise foi obtida com o auxílio do software PAST (PAleontological STatistics) 2.16.

\section{RESULTADOS E DISCUSSÃO}

Durante o período analisado foram capturados um total de 95 ovos e 398 larvas de peixes. As larvas foram classificadas em 25 táxons, pertencentes a 05 ordens, distribuídas em 16 famílias, 19 gêneros e 18 espécies (Tabela 1). Os indivíduos com estruturas danificadas ou em estágio muito inicial de desenvolvimento apresentaram o porcentual de $3,27 \%$ do material coletado.

No geral, os Characiformes apresentaram a maior riqueza de espécies (12) e contribuíram com cerca de $75 \%$ dos indivíduos capturados e identificados, seguido pelos Siluriformes (4), Clupeiformes (1), Perciformes (1) e Gymnotiformes (1), este último teve contribuição inferior a $1 \%$. Dentre os Characiformes, as famílias Hemiodontidae, Characidae, Triportheidae, Prochilodontidae e Anostomidae apresentaram a maior predominância numérica, perfazendo juntas mais de $60 \%$ das larvas capturadas, tornando os espécimes destas famílias os mais representativos na área de estudo.

O principal fator que explica a predominância dos Characiformes é a estratégia reprodutiva de algumas espécies do grupo, principalmente as que realizam movimentos migratórios como os pacus, aracus, sardinhas, branquinhas, flexeiras e curimatãs, que possuem fecundação externa e desova total, contribuindo com a elevada quantidade de ovos e larvas derivando nos rios da bacia amazônica, durante a elevação do nível da água (LIMA; ARAÚJO-LIMA, 2004). No entanto, é comum a baixa ocorrência e/ou ausência de algumas espécies nas amostras, devido às características ecológicas de cada grupo ou espécie como, por exemplo, alguns Ciclídeos (Perciformes) que são sedentários, formam casais, geralmente constroem ninhos nas pausadas e pedras nas margens de rios e nos lagos, possuem cuidado parental e contribuem com menor número de descendentes (GOMIERO; BRAGA, 2004), não sendo passíveis de serem capturados com o apetrecho de pesca empregado, uma vez que este foi direcionado para a captura de organismos planctônicos. 
Tabela 1. Densidade das larvas de peixes (larvas. $10 \mathrm{~m}^{-3}$ ) coletadas nas quatro campanhas realizadas (C1 - seca; C2 - enchente; C3 - cheia; e C4 - vazante), entre os anos de 2014 e 2015, na região da Volta Grande do Xingu (PA), município de Senador José Porfírio, Pará

\begin{tabular}{|c|c|c|c|c|c|c|c|c|c|c|c|c|c|c|c|}
\hline \multirow{2}{*}{ Táxons } & \multirow{2}{*}{$\mathbf{N}$} & \multirow{2}{*}{ Ar } & \multirow{2}{*}{ IC } & \multicolumn{4}{|c|}{ Rio Itatá } & \multicolumn{4}{|c|}{ Rio Ituna } & \multicolumn{4}{|c|}{ Rio Xingu } \\
\hline & & & & C1 & C2 & C3 & C4 & C1 & C2 & C3 & C4 & C1 & C2 & C3 & C4 \\
\hline CHARACIFORMES* & 14 & 3,52 & & - & - & $<0,01$ & - & - & - & 0,01 & - & 0,01 & $<0,01$ & - & 0,01 \\
\hline \multicolumn{16}{|l|}{ Anostomidae } \\
\hline Leporinus sp. & 30 & 7,54 & $\dagger$ & - & - & - & - & - & - & - & - & 0,01 & $<0,01$ & 0,01 & 0,03 \\
\hline Schizodon vittatus (Valenciennes, 1850) & 4 & 1,01 & $\dagger$ & - & - & - & - & - & - & $<0,01$ & - & - & - & $<0,01$ & $<0,01$ \\
\hline Characidae ${ }^{\star \star}$ & 32 & 8,04 & & - & - & - & $<0,01$ & $<0,01$ & - & - & $<0,01$ & $<0,01$ & - & 0,02 & 0,01 \\
\hline Hyphessobrycon sp. & 3 & 0,75 & & - & - & - & - & - & - & - & - & - & - & - & $<0,01$ \\
\hline Curimatidae $^{\star *}$ & 24 & 6,03 & & - & - & - & - & - & - & 0,02 & - & 0,02 & - & 0,02 & 0,01 \\
\hline \multicolumn{16}{|l|}{ Erythrinidae } \\
\hline Hoplias malabaricus (Bloch, 1794) & 1 & 0,25 & & - & - & - & - & - & - & $<0,01$ & - & - & - & - & - \\
\hline \multicolumn{16}{|l|}{ Hemiodontidae } \\
\hline Anodus sp. & 1 & 0,25 & $\dagger$ & - & - & - & - & - & - & - & - & - & - & - & $<0,01$ \\
\hline Hemiodus unimaculatus (Bloch, 1794) & 84 & 21,11 & $\dagger$ & $<0,01$ & 0,12 & - & - & - & $<0,01$ & - & - & - & 0,04 & - & - \\
\hline \multicolumn{16}{|l|}{ Prochilodontidae } \\
\hline Prochilodus nigricans Spix \& Agassiz, 1829 & 24 & 6,03 & $\dagger$ & - & - & - & - & - & - & - & - & - & 0,04 & - & - \\
\hline Semaprochilodus cf. brama (Valenciennes, 1850) & 12 & 3,02 & $\dagger$ & - & - & - & - & - & - & - & - & - & 0,02 & - & - \\
\hline Serrasalmidae ${ }^{* \star}$ & 2 & 0,50 & & - & - & - & - & - & - & $<0,01$ & - & - & - & - & - \\
\hline Myloplus rubripinnis (Müller \& Troschel, 1844) & 7 & 1,76 & $\dagger$ & - & $<0,01$ & - & - & - & - & - & - & - & 0,01 & - & - \\
\hline Piaractus brachypomus (Cuvier, 1818) & 1 & 0,25 & $\dagger$ & - & - & - & - & - & - & - & - & - & $<0,01$ & - & - \\
\hline Serrasalmus rhombeus (Linnaeus 1766) & 6 & 1,51 & & - & - & 0,01 & & - & - & $<0,01$ & - & - & - & $<0,01$ & - \\
\hline \multicolumn{16}{|l|}{ Triportheidae } \\
\hline Triportheus spp. & 53 & 13,32 & & - & 0,02 & - & - & - & 0,02 & - & - & 0,01 & 0,04 & - & 0,01 \\
\hline \multicolumn{16}{|l|}{ CLUPEIFORMES } \\
\hline Engraulidae & 44 & 11,06 & & - & 0,06 & - & - & - & - & - & - & 0,02 & $<0,01$ & - & - \\
\hline \multicolumn{16}{|l|}{ Pristigasteridae } \\
\hline Ilisha amazonica (Miranda Ribeiro, 1920) & 1 & 0,25 & & - & - & - & - & - & - & - & - & - & - & - & $<0,01$ \\
\hline \multicolumn{16}{|l|}{ GYMNOTIFORMES } \\
\hline \multicolumn{16}{|l|}{ Apteronotidae } \\
\hline Sternarchella sp. & 2 & 0,50 & & - & - & - & - & - & - & - & - & - & - & $<0,01$ & - \\
\hline \multicolumn{16}{|l|}{ PERCIFORMES } \\
\hline \multicolumn{16}{|l|}{ Sciaenidae } \\
\hline Plagioscion squamosissimus (Heckel, 1840) & 12 & 3,02 & $\dagger$ & - & - & - & - & - & - & - & - & 0,01 & - & - & 0,01 \\
\hline \multicolumn{16}{|l|}{ SILURIFORMES } \\
\hline Auchenipteridae & & & & & & & & & & & & & & & \\
\hline Auchenipterus nuchalis (Spix, 1829) & 1 & 0,25 & & - & - & - & - & - & - & - & - & - & - & $<0,01$ & - \\
\hline Tatia sp. & 13 & 3,27 & & - & - & 0,01 & - & - & - & 0,01 & - & - & - & $<0,01$ & - \\
\hline Cetopsidae & & & & & & & & & & & & & & & \\
\hline Cetopsis coecutiens (Lichtenstein, 1819) & 6 & 1,51 & & - & - & 0,01 & - & - & - & - & - & - & - & - & - \\
\hline Pimelodidae & & & & & & & & & & & & & & & \\
\hline Pimelodus sp. & 7 & 1,76 & & & & & & & & & & - & - & 0,01 & - \\
\hline Trichomycteridae* & 1 & 0,25 & & - & - & - & - & - & - & - & - & - & - & $<0,01$ & - \\
\hline Larvas não identificadas & 13 & 3,27 & & - & - & - & - & - & - & - & - & 0,01 & - & - & 0,01 \\
\hline Total de larvas & 398 & - & & 0,01 & 0,16 & 0,02 & $<0,01$ & 0,01 & 0,02 & 0,03 & $<0,01$ & 0,09 & 0,11 & 0,08 & 0,13 \\
\hline Total de ovos & 95 & - & & - & 0,02 & $<0,01$ & - & - & $<0,01$ & - & - & 0,02 & 0,02 & 0,02 & 0,16 \\
\hline
\end{tabular}

*Espécimes identificados somente em nível de ordem; **Espécimes identificados somente em nível de família; $\mathbf{N}=$ número total de indivíduos; $\mathbf{A r}=$ Abundância relativa; e IC $=$ Importância comercial. Táxons enquadrados segundo Reis et al. (2003) e Oliveira et al. (2011).

Considerando o perfil da composição das assembleias de larvas de peixes capturadas, os grupos de maior abundância e diversidade de espécies estiveram em conformidade com os padrões gerais estabelecidos para a Amazônia (BARLETTA et al., 2010), nos quais os rios apresentam grande densidade de larvas planctônicas, em que os Characiformes, Siluriformes e Clupeiformes representam a maioria das espécies amostradas para estes ambientes, seguida pelos Perciformes e outras ordens (ARAÚJO-LIMA; OLIVEIRA, 1998; LIMA; ARAÚJO-LIMA, 2004; OLIVEIRA; FERREIRA, 2008). 
Dentre os indivíduos capturados foram registrados a presença de diversos espécimes migradores e de interesse comercial, tais como Myloplus rubripinnis (Müller \& Troschel, 1844) (pacu), Prochilodus nigricans Spix \& Agassiz, 1829 (curimatã), Semaprochilodus brama (Valenciennes, 1850) (ariduia), Piaractus brachypomus (Cuvier, 1818) (pirapitinga), Plagioscion squamosissimus (Heckel, 1840) (pescada branca), Triportheus spp. (sardinhas) e diversos engraulídeos (sardinha), curimatídeos (branquinhas), hemiodontídeos (flexeira) e anostomídeos (aracus e piaus). Estes espécimes são importantes para a região, pois desempenham um papel fundamental na transferência de energia, apresentando relevante papel ecológico. Além disso, são considerados uma das principais fontes de renda, alimento da população local e são mais visados pela atividade pesqueira (ISAAC et al., 2015).

Foram registrados indivíduos em todos os estágios de desenvolvimento, com predominância de larvas em fase de pré-flexão (58\%), seguido por flexão (25\%), pósflexão (12\%) e larval vitelino (5\%). A baixa participação da fase larval vitelina deve-se provavelmente ao fato deste se tratar do estágio mais curto do desenvolvimento, que ocorre logo após a desova e eclosão, e que nem sempre é capturado nas amostragens. De acordo com Zacardi, Sobrinho e Silva (2014) a ocorrência de ovos e larvas de peixes no plâncton, em diversos estágios de desenvolvimento e em diferentes épocas do ano, é extremamente variável, estando tais variações relacionadas, principalmente, ao ciclo anual de maturação gonadal diferenciado das espécies, levando a mudanças na distribuição e na composição quali-quantitativa do ictioplâncton.

Entretanto, a presença desse estágio, em conjunto com as elevadas abundâncias de larvas em fase de pré-flexão, sugere que as áreas de coleta estão próximas aos sítios de reprodução e caracterizam a região da Volta Grande como áreas de retenção e criação para diversas espécies de peixes. Essas informações indicam a presença de atividade reprodutiva ao longo desses rios, enfatizando a importância destes ambientes para a manutenção dos estoques pesqueiros, bem como a necessidade de se adotar ações de manejo visando a preservação dos estoques naturais.

A ANOVA quando aplicada aos dados gerais de densidade de ovos $(\mathrm{F}=2,88$; $p=0,014)$ e de larvas $(\mathrm{F}=4,24 ; p=0,001)$ apresentou diferenças significativas, entre as estações de amostragem, evidenciando uma variação espacial. $\mathrm{O}$ teste de 
Tukey confirmou diferenças significativas entre os trechos de rios $(p<0,05)$, com picos de maior densidade de larvas nos tributários Itatá e Ituna, quando comparadas com as estações de amostragens localizadas ao longo do rio Xingu (Figura 4), com destaque para as estações E01 e E06. Entretanto, as estações E14 e E15, próximas à área de pedral e banco de areia no rio Xingu, apresentaram as maiores densidades de ovos capturados, juntamente com áreas mais internas dos tributários (E03 e E06, respectivamente).

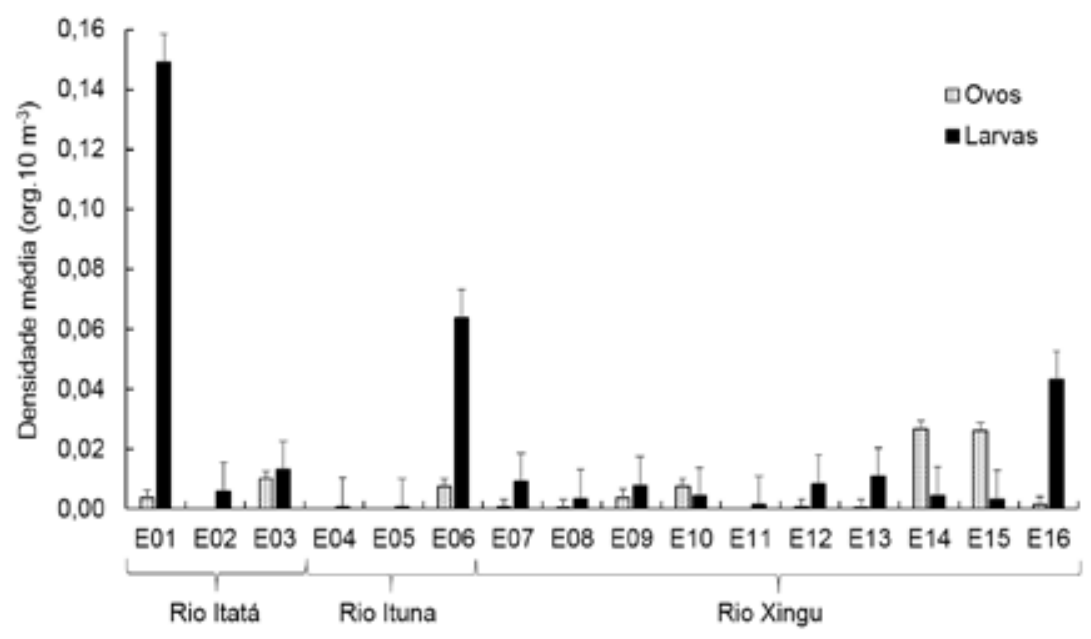

Figura 4. Variação espacial e erro padrão da densidade do ictioplâncton capturado nas estações de amostragem ao longo da Volta Grande do Xingu (PA), nas quatro campanhas realizadas entre os anos de 2014 e 2015.

A elevada concentração de larvas capturadas na estação E16 ao longo do rio Xingu demonstra a importância das áreas de bocas de canais de lago para o desenvolvimento das larvas que utilizam estes ambientes como locais de dispersão. Esse agrupamento próximo às futuras áreas de inundação pode se tratar de uma estratégia larval, uma vez que algumas espécies da ictiofauna amazônica utilizam os ambientes lacustres como áreas de abrigo e forrageio, durante as fases iniciais de desenvolvimento, favorecendo desta maneira a sobrevivência da prole (LEITE; ARAÚJO-LIMA, 2002). Sugere-se que estas regiões de confluência de lagos e rios funcionem como importantes áreas de estímulo para desova desses peixes e indicam o uso destes habitats como zona de colonização e dispersão larval. 
No geral, a baixa densidade de ovos em relação às larvas pode ter sido influenciada pelo curto espaço de tempo entre a incubação e a eclosão, como verificado por Araújo-Lima e Oliveira (1998), pois este tempo para a maioria das espécies que dispersam ovos e larvas pelas correntes dos rios é relativamente curto entre 12 a 18 horas (NAKATANI et al., 2001), tendo para os ovos menor tempo de exposição no ambiente do que para as larvas (KIPPER, 2010).

Entretanto, os resultados demostram que os sítios de desova de inúmeras espécies ocorrem próximos aos bancos de areia situados no trecho do rio Xingu e na parte mais interna dos tributários que apresentam extensas faixas de floresta marginal inundável que minimiza a radiação incidente sobre a superfície aquática, fornece fontes alimentares e serve como refúgio e proteção contra predadores, indicando que estes locais, provavelmente, possuem melhores condições para a desova.

Os resultados também evidenciam diferença significativa na variação sazonal da densidade de ovos ( $\mathrm{F}=2,62 ; \mathrm{p}=0,016)$ e larvas $(\mathrm{F}=3,00 ; p=0,020)$, com as maiores contribuições de ictioplâncton registradas, durante a segunda campanha (C2 $-p<0,05)$, coincidindo com o período de elevação do nível fluviométrico do rio Xingu (Figura 5). As demais campanhas (C1, C3 e C4) não apresentaram diferença significativa pelo teste de Tukey ( $p<0,27, p<0,16$ e $p<0,12$, respectivamente).

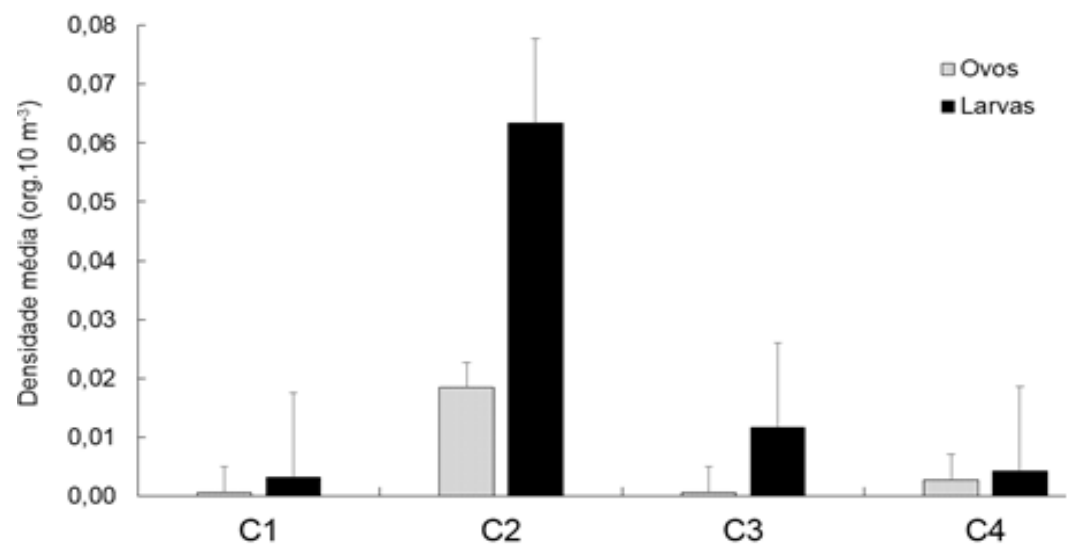

Figura 5. Variação temporal e erro padrão da densidade do ictioplâncton capturado durante as campanhas (C1 - seca; C2 - enchente; C3 - cheia; e C4 - vazante), ao longo da Volta Grande do Xingu (PA). 
A maior captura de ovos e larvas de peixes na enchente confirma este período como o de maior atividade reprodutiva dos peixes neste trecho do médio rio Xingu que, segundo Ghilardi-Júnior e Camargo (2009), nesta área a atividade reprodutiva se estende de dezembro a maio. A reprodução de espécies de peixes migradores, como os Characiformes, está vinculada ao ciclo anual de chuvas e ao aumento do nível hidrológico, sendo estes os fatores que desempenham o papel mais importante na reprodução destes peixes na área de estudo (CAMARGO; LIMAJÚNIOR, 2007).

A principal diferença do setor médio do rio Xingu em relação aos rios da planície amazônica consiste na limitada área de inundação nas margens do rio, que apresentam geomorfologia ondulada e com leito rochoso que se expande ou contrai em função do regime de cheias, garantindo não só o sucesso reprodutivo, mas o acesso a fontes alóctones de origem vegetal fornecendo alimento para os peixes (GIARIZZO; CAMARGO, 2009).

Muitas espécies de peixes apresentam adaptação na migração vertical na coluna d'água que lhe conferem maior segurança e probabilidade de sobrevivência. Estes mecanismos consistem na submersão das larvas durante o dia e emersão na coluna d'água durante a noite. Essa estratégia no comportamento noturno auxilia a diminuição da competição por espaço e alimento, aumentando as possibilidades de sobrevivência e garantindo, desta forma, sucesso no recrutamento (ZACARDI et al., 2015).

Neste sentido, nossos dados exibiram uma alta abundância relativa, com contribuição de $82,11 \%$ dos ovos e $92,10 \%$ das larvas, durante o período noturno, entretanto, não foi registrada diferença significativa em relação à variação circadiana dos ovos e nem de larvas coletadas $(p>0,05)$. As maiores abundâncias de ovos e larvas no período noturno indicam desova ao entardecer e maior atividade das larvas à noite, em função da busca por alimento (BAUMGARTNER et al., 2004), fuga de predadores visuais e desorientação noturna (BAUMGARTNER, 2001).

As desovas efetuadas durante o entardecer são regra geral para a maioria das espécies de peixes tropicais, que se aproveitam da baixa luminosidade e dos horários de elevada temperatura da água para realizarem suas desovas (GRAFF, 1999). De acordo com Humphries, King e Koehn (1999) as variáveis como o fotoperíodo, 
a hidrodinâmica do rio e a temperatura da água são essenciais na ocorrência, densidade e crescimento dos peixes nos primeiros estágios de vida.

Em relação às variáveis que contribuem para o desenvolvimento inicial dos peixes, os dois primeiros eixos da Análise de Componentes Principais do rio Itatá explicaram 95,13\%, do Ituna 95,93\% e do Xingu 94,52\% da variabilidade dos dados. No rio Itatá, as variáveis temperatura, pH e oxigênio dissolvido contribuíram positivamente na ordenação do PC1 e o inverso foi observado para o índice pluviométrico e o nível fluviométrico. Para o rio Ituna, o índice pluviométrico e o nível fluviométrico contribuíram positivamente no PC1 e a condutividade elétrica no PC2, entretanto, a temperatura, o pH e o oxigênio dissolvido apresentaram-se negativos no PC1. O rio Xingu teve contribuição positiva dos dados fluviométricos e pluviométricos no PC1 e o inverso foi observado para a temperatura e oxigênio dissolvido. Neste caso, o pH foi o único parâmetro que contribuiu positivamente no PC2 (Tabela 2).

Tabela 2. Autovetores obtidos através da Análise de Componentes Principais e autovalores dos eixos retidos para interpretação

\begin{tabular}{ccccccc}
\hline \multirow{2}{*}{ Variáveis ambientais } & \multicolumn{2}{c}{ Rio Itatá } & Rio Ituna & \multicolumn{2}{c}{ Rio Xingu } \\
\cline { 2 - 7 } & PC1 & PC2 & PC1 & PC2 & PC1 & PC2 \\
\hline Temperatura & 0,989 & $-0,064$ & $-0,889$ & $-0,413$ & $-0,952$ & $-0,182$ \\
Oxigênio Dissolvido & 0,940 & $-0,137$ & $-0,966$ & $-0,028$ & $-0,972$ & 0,100 \\
pH & 0,978 & 0,066 & $-0,978$ & 0,097 & $-0,129$ & 0,977 \\
Condutividade elétrica & $-0,111$ & 0,992 & 0,014 & 0,988 & 0,932 & $-0,204$ \\
Nível fluviométrico & $-0,940$ & $-0,083$ & 0,910 & $-0,330$ & 0,914 & 0,291 \\
Índice pluviométrico & $-0,974$ & $-0,163$ & 0,991 & $-0,014$ & 0,988 & $-0,026$ \\
\hline
\end{tabular}

Analisando a ordenação dos dados em ambos os rios observa-se que a plotagem dos dois eixos agrupou as estações de amostragem por campanha evidenciando um padrão sazonal, em que a elevada abundância de ovos e larvas esteve relacionada ao pulso de inundação, devido à grande contribuição da pluviosidade local e do nível crescente das águas do rio Xingu. As variáveis de oxigênio dissolvido, 
condutividade elétrica, $\mathrm{pH}$ e temperatura também influenciam diferentemente, com maior ou menor grau, a ocorrência e distribuição do ictioplâncton em cada rio analisado (Figura 6).

As correlações de Pearson realizadas não foram significativas para a fase do ciclo hidrológico de vazante e também não mostraram um padrão claro de distribuição espacial entre os parâmetros e a ocorrência do ictioplâncton em nenhum rio analisado.

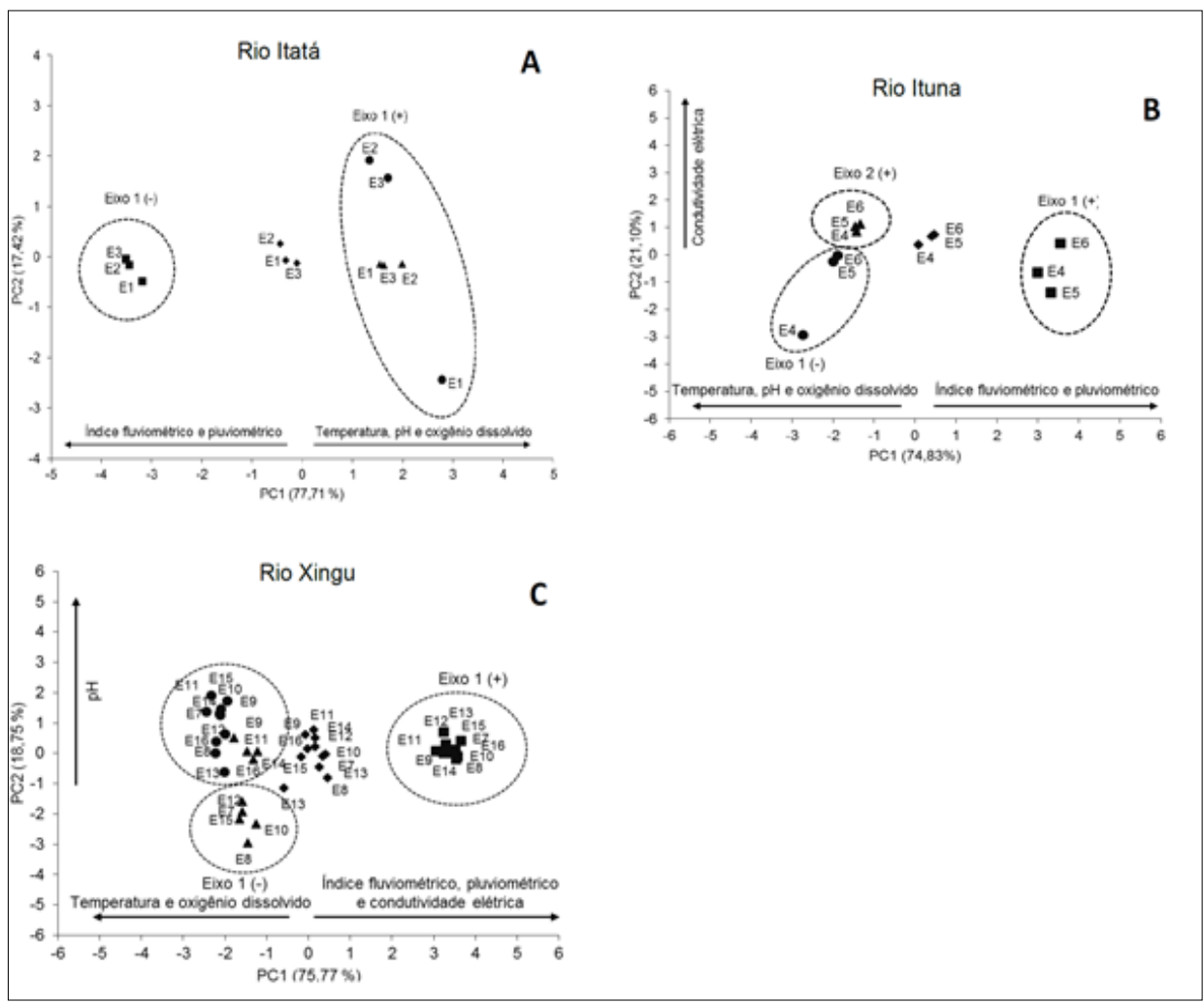

Figura 6. Resultado gráfico entre os eixos da Análise de Componentes Principais (ACP), plotados a partir do logaritmo das variáveis abióticas obtido nas estações de amostragem nos rios Itatá (E1, E2 e E3), Ituna (E4, E5 e E6) e Xingu (E7, E8, E9, E10, E11, E12, E13, E14, E15 e E16), durante as campanhas de amostragem: $\mathrm{C} 1=\boldsymbol{\Delta}, \mathrm{C} 2=\boldsymbol{\bullet}, \mathrm{C} 3=\boldsymbol{\square}$ e $\mathrm{C} 4=\diamond$.

Os índices de $\mathrm{pH}$ mais baixos foram registrados durante a primeira campanha (período de seca do rio Xingu), provavelmente devido à influência de grande quantidade de matéria orgânica alóctone nas áreas marginais do canal, concentradas 
na calha dos rios devido ao menor nível de água, que é comum no período de setembro. $\mathrm{O}$ pH é altamente influenciado pela quantidade de matéria orgânica a ser decomposta, pois quanto maior a quantidade disponível menor é o pH devido à formação de ácidos húmicos, podendo variar dependendo da área. Vale ressaltar que em ambientes onde a ação antrópica é marcante, a qualidade da água é afetada não só por fatores naturais, mas também por impactos das atividades humanas.

O regime de chuvas no Brasil apresenta sazonalidade marcante com estação seca e chuvosa em épocas diferentes do ano de acordo com a localização geográfica e isso pode afetar as concentrações das variáveis físico-químicas nos rios, já que a precipitação pluviométrica possui uma relação direta com a biomassa fitoplanctônica, material em suspensão e o teor de oxigênio dissolvido, e relação inversa com a temperatura e transparência da água (BASTOS; FEITOSA; MUNIZ, 2005).

Essa variabilidade sazonal é que faz com que as condições do meio se tornem favoráveis à desova, sendo responsáveis pelo sincronismo e distribuição de ovos e de larvas de peixes. Como os ovos e as larvas derivam, sua sobrevivência depende da disponibilidade de habitats como as áreas de capoeira, florestas e ilhas fluviais que sofrem influência do pulso de inundação e da conectividade longitudinal e lateral para estas áreas neste trecho do rio Xingu. E, dessa forma, ampliam-se as probabilidades de refúgio e exploração de novas fontes alimentares, maximizando as chances de desenvolvimento e sobrevivência dos peixes durante as primeiras fases do seu ciclo de vida.

A magnitude, a frequência, a duração e a previsibilidade do pulso de inundação são as características mais importantes na regulação dos processos ecológicos dos peixes (BUNN; ARTHINGTON, 2002; PRADO, 2014). Apesar disso, a construção de um represamento ou reservatório altera o regime hidrológico natural do rio e regulariza a vazão dos trechos a jusante de uma barragem (HUMPHRIES; SERAFINE; KING, 2002), compromete a conectividade do rio com as áreas alagáveis, altera a qualidade da água e bloqueia as rotas migratórias (AGOSTINHO et al., 2007), sendo estes os principais fatores negativos à reprodução das espécies de peixes migradores (ESGUÍCERO; ARCIFA, 2010; GANDINI et al., 2012).

Além de modificar as condições apropriadas para a reprodução e/ou das condições necessárias ao desenvolvimento das larvas (HUMPHRIES; LAKE, 2000), 
afetando as migrações reprodutivas ascendentes dos adultos, como também a migração descendente realizada pelo ictioplâncton (AGOSTINHO et al., 2007).

Portanto, a estrutura da assembleia ictioplanctônica do médio rio Xingu é influenciada por vários fatores intrínsecos e extrínsecos como os diferentes parâmetros ambientais, corpos hídricos ou habitats, pois estes podem ser utilizados por inúmeras espécies, sobretudo aquelas de interesse econômico regional, para completar seu ciclo de vida, independente da sua fase de desenvolvimento, confirmando assim a relevância de se manter a integridade do trecho da Volta Grande do Xingu, já que os dados confirmaram a região como importante área para a conservação, preservação e manutenção de recursos pesqueiros locais.

\section{CONSIDERAÇÕES FINAIS}

As amostragens realizadas no trecho médio do rio Xingu revelam a importância deste ambiente para a manutenção dos estoques pesqueiros da região, devido à quantidade de ictioplâncton capturado na calha do rio principal, em locais próximos a bancos de areia, boca de lagos e ao longo dos tributários, sendo possível sugerir que o trecho da Volta Grande do Xingu e seus tributários são utilizados como área de desova, criação e dispersão de ovos e larvas de uma variedade de espécies de peixes, revelando a importância de se manter este ambiente em suas condições mais próximas do natural e do papel relevante na conservação da biodiversidade regional.

Dessa forma, as informações apresentadas sobre as assembleias ictioplanctônicas no trecho do médio rio Xingu são de fundamental importância e devem ser consideradas para identificar potencialidades, limitações, dinâmicas ecológicas e importância relativas dos corpos d'água e seus habitats, no recrutamento das principais espécies da ictiofauna regional, especialmente aquelas de maior interesse econômico, que podem ser mais bem manejadas e utilizadas sustentavelmente a partir do subsídio destas informações. 


\section{AGRADECIMENTO}

Ao Dr. Frank Raynner Vasconcelos Ribeiro, aos Mes. André Luiz Colares Canto e Carlison Silva de Oliveira (Coleção Ictiológica - Universidade Federal do Oeste do Pará), pelo auxílio na identificação de alguns grupos de peixes. À Belo Sun Mineração Ltda. por todo o apoio logístico.

\section{REFERÊNCIAS}

AGOSTINHO, A. A.; JÚLIO-JÚNIOR, H. F.; BORGHETTI, J. R. Considerações sobre os impactos dos represamentos na ictiofauna e medidas para sua atenuação. Um estudo de caso: reservatório de Itaipu. Revista UNIMAR, v. 14, p. 89-107, 1992.

AGOSTINHO, A. A.; MARQUES, E. E.; AGOSTINHO, C. S.; ALMEIDA, D. A.; OLIVEIRA, R. J.; RODRIGUES, J. B. M. Fish ladder of Lajeado Dam: migration on one way routes? Neotropical Ichthyology, v. 5, p. 121-130, 2007.

AGOSTINHO, A. A.; PELICICE, F. M.; GOMES, L. C. Dams and the fish fauna of the Neotropical region: impacts and management related to diversity and fisheries. Brazilian Journal of Biology, v. 68, n. 4, p. 1119-1132, 2008.

ARAÚJO-LIMA, C. A. R. M. Egg size and larval development in Central Amazon fish. Journal of Fish Biology, v. 44, p. 371-389, 1994.

ARAÚJO-LIMA, C. A. R. M.; DONALD, E. Número de vértebras de Characiformes e seu uso na identificação de larvas do grupo. Acta Amazonica, v. 18, n. 1, p. 351-358, 1988.

ARAÚJO-LIMA, C. A. R. M.; OLIVEIRA, E. C. Transport of larval fish in the Amazon. Journal of Fishes Biology, v. 53, p. 297-306, 1998.

BARLETTA, M.; JAUREGUIZAR, A. J.; BAIGUN, C.; FONTOURA, N. F.; AGOSTINHO, A. A.; ALMEIDA-VAL, V. M. F.; VAL, A. L.; TORRES, R. A.; JIMENES-SEGURA, L. F.; GIARRIZZO, T.; FABRÉ, N. N.; BATISTA, V. S.; LASSO, C.; TAPHORN, D. C.; COSTA, M. 
F.; CHAVES, P. T.; VIEIRA, J. P.; CORRÊA, M. F. M. Fish and aquatic habitat conservation in South America: a continental overview with emphasis on neotropical systems. Journal of Fish Biology, v. 76, n. 9, p. 2118-2176, 2010.

BASTOS, R. B.; FEITOSA, F. A. N.; MUNIZ, K. Variabilidade espaço-temporal da biomassa fitoplanctônica e hidrologia do rio Una (Pernambuco - Brasil). Tropical Oceanography, v. 33, n. 1, p. 1-18, 2005.

BAUMGARTNER, G.; NAKATANI, K.; GOMES, L. C.; BIALETZKI, A.; SANCHES, P. V.; MAKRAKIS, M. C. Identification of spawning sites and natural nurseries of fishes in the Upper Paraná River, Brazil. Environmental Biolog y of Fishes, v. 71, p. 115-125, 2004.

BAUMGARTNER, G. Determinação dos locais de desova e criadouros naturais de peixes e influência dos fatores abióticos sobre a abundância de larvas no alto Rio Paraná, Brasil. 2001. 62 f. Tese (Doutorado em Ecologia de Ambientes Aquáticos Continentais) - Universidade Estadual de Maringá, Maringá, PR.

BENEDITO-CECILIO, E.; AGOSTINHO, A. A. Distribution, abundance and use of different environments by dominant ichthyofauna in the influence area of the Itaipu Reservoir. Acta Scientiarum, v. 22, n. 2, p. 429-437, 2000.

BUNN, S. E.; ARTHINGTON, A. H. Basic principles and ecological consequences of altered flow regimes for aquatic biodiversity. Environmental Management, v. 30, p. 492-507, 2002.

CAMARGO, M. A Comunidade íctica e suas interrelações tróficas como indicadoras de integridade biológica na Área de Influência do Projeto Hidrelétrico Belo Monte - Rio Xingu-PA. 2004. 167f. Tese (Doutorado em Zoologia) - Universidade Federal do Pará, Departamento de Ciências Biológicas, Belém, 2004.

CAMARGO, M.; LIMA JUNIOR, W. M. A. Aspectos da biologia reprodutiva de seis espécies de peixes de importância comercial do médio Rio Xingu - Bases para seu manejo. Uakari, v. 3, n. 1, p. 64-77, 2007. 
CAMARGO, M.; GONÇALVES, A. P.; CARNEIRO, C. C.; NISA E CASTRO, G. T. Pesca de consumo. In: CAMARGO, M.; GHILARDI-JÚNIOR, R. (Eds.). Entre a terra as águas e os pescadores do médio rio Xingu: uma abordagem ecológica. Belém: Eletronorte, 2009, p. 265-282.

CAMARGO, M.; GHILARDI, R. Entre a terra as águas e os pescadores do médio rio Xingu: uma abordagem ecológica. Belém: Eletronorte, 2009, 329p.

CARVALHO JÚNIOR, J. R.; ZACARDI, D. M.; BITTENCOURT, S. C. S.; BEZERRA, M. F. C.; NUNES, J. L. G.; NAKAYAMA, L. Apetrechos de pesca ornamental utilizados pelos juruna da terra indígena Paquiçamba (Pará, Brasil). Boletim Técnico-Científico do CEPNOR, v. 11, n. 1, p. 71-79, 2011.

ESGUÍCERO, A. L. H.; ARCIFA, M. S. Fragmentation of a Neotropical migratory fish population by a century-old dam. Hydrobiologia, v. 638, n. 1, p. 41-53, 2010.

GANDINI, C. V.; BORATTO, I. A.; FAGUNDES, D. C.; POMPEU, P. S. Estudo da alimentação dos peixes no rio Grande à jusante da usina hidrelétrica de Itutinga, Minas Gerais, Brasil. Iheringia, Série Zoologia, v. 102, n. 1, p. 56-61, 2012.

GHILARDI-JÚNIOR, R.; CAMARGO, M. 2009. Breve visão do Xingu. In: CAMARGO, M.; GHILARDI-JÚNIOR, R. (Ed.). Entre a terra, as águas e os pescadores do médio rio Xingu: uma abordagem ecológica. Belém: Eletronorte, 2009, p. 17-32.

GIARRIZZO, T.; CAMARGO, M. As piracemas. In: CAMARGO, M.; GHILARDI-JÚNIOR, $\mathrm{R}$. (Ed.). Entre a terra as águas e os pescadores do médio rio Xingu: uma abordagem ecológica. Belém: Eletronorte, 2009, p. 283-296.

GOMIERO, L. M.; BRAGA, F. M. S. Reproductive of species of the genus Cichla in a reservoir in Southeastern Brazil. Brazilian Journal Biolog y, v. 64, n. 3, p. 613-624, 2004.

GRAAF, G. J.; BORN, A. F.; UDDIN, A. M. K.; HUDA, S. Larval fish movement in the river Lohajang, Tangail, Bangladesh. Fisheries Management and Ecology, v. 6, p. 109-120, 1999. 
HUMPHRIES, P.; KING, A. J.; KOEHN, J. D. Fish, flows and flood plains: links between freshwater fishes and their environment in the Murray Darling River system, Australia. Environmental Biology of Fishes, v. 56, p. 129-151, 1999.

HUMPHRIES, P.; LAKE, P. S. Fish larvae and management of regulated rivers. Regulated Rivers: Research \& Management, v. 16, p. 421-432, 2000.

HUMPHRIES, P.; SERAFINE, L. G.; KING, A. J. River regulation and fish larvae: variation through space and time. Freshwater Biology, v. 47, p. 1307-1331, 2002.

ISAAC, V. J.; ALMEIDA, M. C.; CRUZ, R. E. A.; NUNES, L. G. Artisanal fisheries of the Xingu River basin in Brazilian Amazon. Brazilian Journal of Biology, v. 75, n. 3, p. 125-137, 2015.

JACKSON, D. A. Stopping rules in principal components analysis: a comparsion of heuristical and statistical approaches. Ecology, v. 74, p. 2204-2214, 1993.

JAGER, H. I.; CHANDLER, J. A.; LEPLA, K. B.; WINKLE, W. V. A theoretical study of river fragmentation by dams and its effects on white sturgeon populations. Environmental Biology of Fishes, v. 60, p. 347-361, 2001.

KIPPER, D. Distribuição do ictioplâncton no reservatório de Rosana, rio Paranapanema, Brasil. 2010. 56 f. Dissertação (Mestrado em Ciências Ambientais) Universidade Estadual de Maringá. Departamento de Biologia, Maringá, 2010.

LEITE, R. G.; ARAÚJO-LIMA, C. A. R. M. Feeding of the Brycon cephalus, Triportbeus elongatus and Semaprochilodus insignis (Osteichthyes, Characiformes) larvae in Solimões/Amazonas River and floodplain areas. Acta Amazonica, v. 32, n. 3, p. 129$147,2002$.

LEITE, R. G.; CANÃS, C.; FORSBERG, B.; BARTHEM, R.; GOULDING, M. Larvas dos grandes bagres migradores. Instituto Nacional de Pesquisa da Amazônia / Asociación para la Conservación de la Cuenca Amazónica, 2007, 127 p.

LIMA, A. C.; ARAÚJO-LIMA, C. A. R. M. The distribution of larval and juvenile fishes in Amazonian rivers of different nutrient status. Freshwater Biology, v. 49, p. 1-14, 2004 . 
LUCAS, E. W. M.; SOUSA, F. A. S.; SILVA, F. D. S.; LUCIO, P. S. Modelagem hidrológica determinística e estocástica aplicada à região hidrográfica do Xingu - Pará. Revista Brasileira de Meteorologia, v. 24, n. 3, p. 308-322, 2009.

MEDEIROS, P. R. P. Influência das Oscilações das Marés na Concentração de Nutrientes e do Plâncton no Estuário do Rio São Francisco. In: PROGRAMA de Pesquisa e Desenvolvimento P\&D para o ciclo 2003/2004. Contrato CTI 92.2005.7480.000. Maceió, 2006.

NASCIMENTO, F. L.; ARAUJO-LIMA, C. A. R. M. Descrição das larvas de Psectrogaster amazonica e Potamorbina altamazonica (Curimatidae, Pisces) da Amazônia Central. Acta Amazonica, v. 23, n. 4, p. 457-472, 1993.

NASCIMENTO, F. L.; ARAÚJO-LIMA, C. A. R. M. Descrição de larvas das principais espécies de peixes utilizadas pela pesca no Pantanal. Corumbá: Embrapa Pantanal, 2000, 25 p.

NAKATANI, K.; AGOSTINHO, A. A.; BAUMGARTNER, G.; BIALETZKI, A.; SANCHES, P. V.; MAKRAKIS, M. C.; PAVANELLI, C. S. Ovos e larvas de peixes de água doce: desenvolvimento e manual de identificação. Maringá: EDUEM, 2001, 378 p.

OLIVEIRA, C.; AVELINO, G. S.; ABE, K.; MARIGUELA, T. C.; BENINE, R. C.; ORTÍ, G.; VARI, R. P.; CASTRO, R. M. C. Phylogenetic relationships within the speciose family Characidae (Teleostei: Ostariophysi: Characiformes) based on multilocus analysis and extensive ingroup sampling. BMC Evolutionary Biology, v. 11, p. 1-25, 2011.

OLIVEIRA, E. C.; FERREIRA, E. J. G. Spawning areas, dispersion and microhabitats of fish larvae in the Anavilhanas Ecological Station, rio Negro, Amazonas State, Brazil. Neotropical Ichthyology, v. 6, n. 4, p. 559-566, 2008.

PRADO, R. C. C. Dinâmica da migração e reprodução dos peixes na região da foz do rio Abaeté, Alto São Francisco, MG. 2014. 93 f. Dissertação (Mestrado em Ecologia e conservação de paisagens fragmentadas e agrossistemas) - Universidade Federal de Lavras, Lavras, 2014.

PETERS, R. K. The role of prediction in limnology. Limnology and Oceanography, 
v. 31, p. 1143-1159, 1986.

REIS, R. E.; KULLANDER, S. O.; FERRAIS-Jr., C. J. Check list of the freshwater fishes of South and Central America. Porto Alegre: EDIPUCRS, 2003, 729p.

SALOMÃO, R. P.; VIEIRA, I. C. G.; SUEMITSU, C.; ROSA, N. A.; ALMEIDA, S. S.; AMARAL, D. D.; MENEZES, M. P. M. As florestas de Belo Monte na grande curva do rio Xingu, Amazônia Oriental. Boletim do Museu Paraense Emílio Goeldi. Ciências Naturais, v. 2, n. 3, p. 57-153, 2007.

ZACARDI, D. M.; SOBRINHO, A. F.; SILVA, L. M. A. Composição e distribuição de larvas de peixes de um afluente urbano na foz do rio Amazonas, Brasil. Actapesca, v. 2, n. 2, p. 1-16, 2014.

ZACARDI, D. M. Variação e abundância do ictioplâncton em canais de maré no Extremo Norte do Brasil. Biota Amazônia, v. 5, n. 1, p. 43-52, 2015.

Recebido em: 31 de agosto de 2015 Revisado em: 21 de dezembro de 2015 Aceito em: 21 de janeiro de 2016 